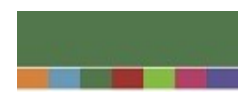

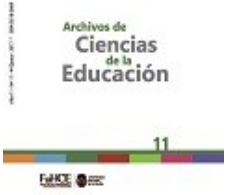

Eara o:
Archivos de Ciencias de la Educación, Vol. 11, n 11, 2016, e024. ISSN 2346-8866

Universidad Nacional de La Plata.

Facultad de Humanidades y Ciencias de la Educación.

Departamento de Ciencias de la Educación.

\title{
La construcción del ingreso como política de inclusión en la Universidad Nacional de La Plata, 1995-2015
}

\author{
The desing of university admission as a policy of inclusion in \\ Humanities of La Plata, 1995-2015
}

\section{Jesica Montenegro}

Universidad Nacional de La Plata, Argentina | montenegro jessica@yahoo.com.ar

\section{PALABRAS CLAVE RESUMEN}

Universidad

Políticas de ingreso

Estrategias institucionales

Humanidades

\section{KEYWORDS}

University

Admission policies

Institutional strategies

Humanities
Este artículo describe y analiza la construcción de las estrategias de ingreso en la Facultad de Humanidades y Ciencias de la Educación de la Universidad Nacional de La Plata durante el período 1995-2015. El trabajo reconstruye las preocupaciones que dieron origen a esas propuestas, sus características y los cambios que han realizado durante el recorte temporal efectuado. Asimismo, analiza la relación de los lineamientos formulados a nivel central de la Universidad con su expresión en la Facultad estudiada, y recupera las perspectivas que sustentan los actores institucionales, los acuerdos y tensiones suscitados con relación al procesamiento de esa política. La investigación aborda la generación y la dinámica de procesamiento de una política de inclusión universitaria, específicamente de ingreso, en la que intervienen en su elaboración actores institucionales con perspectivas singulares.

\section{ABSTRACT}

This article describes and analyses the admission strategies at the School of Humanities and Social Sciences, National University of La Plata from 1995 to 2015. This work reconstructs the main concerns that lead to these proposals, its characteristics and the changes made, throughout the period analyzed. Likewise, it analyses the relationship between the guidelines established at the central level of the university and the form it adopted at the School level studied, while at the same time, it retrieves the institutional actors' viewpoints, the agreements and tensions caused in relation to that policy processing. This research addresses the generation and processing dynamics of an inclusion policy, specifically of admission, in a scenario with institutional actors bearing different points of view. 


\section{Introducción}

En este artículo se describe y analiza la construcción de las estrategias de ingreso en la Facultad de Humanidades y Ciencias de la Educación (FaHCE) de la Universidad Nacional de La Plata (UNLP), durante el período 1995-2015.

El abordaje se enmarca en una investigación más amplia $\underline{2}$ cuyo objetivo fue analizar y comprender la política de acceso a la UNLP y sus formas de procesamiento en las Unidades académicas (UA) por medio de las “estrategias de ingreso" $\underline{3}$ desarrolladas ad hoc, desde el análisis de las relaciones de poder entre los actores intervinientes en cada caso, para el período 1995-2015. El recorte temporal efectuado obedeció al interés por indagar qué cambios introdujo la aprobación de una nueva normativa nacional: la Ley de Educación Superior (LES), sancionada en 1995 y que no sufrió modificaciones hasta el año 2015. Se partió del supuesto que la modificación del marco normativo nacional en 1995 trajo como consecuencia el despliegue de nuevas regulaciones y de allí, el interés por comprender qué incidencia tuvieron esas definiciones en las políticas elaboradas a nivel local con respecto al acceso en la UNLP y su expresión en las estrategias institucionales de distintas UA elegidas para la pesquisa. $\stackrel{4}{ }$

A partir de la sanción de la LES y hasta el período de nuestro estudio, el Estado Nacional dejó en manos de cada institución la potestad para establecer de manera autónoma el régimen de admisión, permanencia y promoción de sus estudiantes. Cabe destacar que antes de la sanción de la LES, cada universidad establecía su régimen de admisión, siendo esto una cuestión de hecho, en razón de la autonomía universitaria que gozaban. Sin embargo, en el marco regulatorio de los años '90, la novedad no sólo refirió a la explicitación de aquélla potestad, sino que además estableció que cada institución que posea más de 50.000 estudiantes definiera su política de ingreso otorgando la posibilidad de que cada Facultad estableciera su propio régimen de admisión. De esta manera, el ingreso a la universidad argentina, tal como lo dispuso la LES, estaba regulado por las propias instituciones del sistema universitario. En tal sentido, las universidades alcanzaron mayor autonomía para regular el acceso de los estudiantes, por lo que la LES habilitó la existencia de una amplia heterogeneidad de estrategias y políticas institucionales (interinstitucional), que incluso se encontraban diferenciadas por unidades académicas y/o carreras (intrainstitucional). En efecto, diversas investigaciones inscriptas en el campo de estudios de la educación superior, caracterizaron específicamente a los sistemas de ingreso a la universidad en nuestro país a partir de los lineamientos de la LES establecidos en 1995. En términos generales, esos estudios mostraron la coexistencia de modalidades de ingreso en las universidades públicas en Argentina, que abarcaban tanto modalidades eliminatorias como irrestrictas (Chiroleu, 1999; Duarte, 2005; Ramallo y Sigal, 2010). Hasta el año 2015, la UNLP quedó comprendida entre tales criterios, dando lugar a la coexistencia -dentro de sus UA-, de estrategias de ingreso muy disímiles en sus propósitos y modalidades, participando en su elaboración diversos actores con perspectivas singulares. La autonomía de las facultades para establecer las estrategias de ingreso condujo a la configuración de una política de acceso descentralizada y derivó en el diseño y desarrollo de estrategias con características particulares y con diversas direccionalidades que variaron en un abanico de opciones y formas, desde el carácter introductorio hasta la restricción (Bracchi, Sannuto y Mendy, 2002).

Es preciso señalar que UA analizada se organiza bajo una lógica disciplinar (Clark, 1983), brinda 
treinta carreras de grado, contando con un total de diez departamentos docentes y tiene quince unidades de investigación. Posee una matrícula total de 10.051 estudiantes y 2.463 ingresantes. $\underline{5}$ Estos indicadores permiten advertir que el tamaño y la complejidad (Balgridge et al, 1973) de esta UA es grande. El campo académico disciplinar abarca tanto "disciplinas blandas puras" como “disciplinas blandas aplicadas”, siguiendo la categorización propuesta por Becher (2001). Cuenta con gran cantidad de carreras de tamaño diverso - pequeñas, medianas y grandes - considerando las variaciones que se registran en sus matrículas de ingresantes. ${ }^{6}$ Se distingue una gran heterogeneidad de estrategias de ingreso configuradas por cada departamento docente dentro de los lineamientos de la política de ingreso de la Institución. La mayoría de las estrategias de ingreso se caracterizan por ser de carácter “orientador” e “introductorio”. La modalidad de ingreso es irrestricto con cursos obligatorios u optativos, según las carreras. Algunos departamentos docentes optaron por establecer un sistema de admisión mediante “evaluación sin prueba”, aunque el Departamento de Lenguas y Literaturas Modernas, como veremos más adelante, optó por un sistema de "evaluación con prueba”, instancia que define el inicio o no de las cursadas de las materias específicas del primer año, aunque sin restricción para las asignaturas curriculares en castellano como alumnos regulares.

Las perspectivas organizacionales (Clark, 1983; Becher, 2001) y la sociología de la cultura de Bourdieu (2003; 2008), muestran que las universidades poseen niveles de autonomía relativa respecto del poder central por sus características organizacionales. De este modo, las universidades desarrollan dinámicas específicas por la intervención de los actores académicos que modelan su ambiente de trabajo e inciden en el procesamiento de los cambios. Los aportes de los enfoques neoinstitucionalistas (Cox, 1993; Brint y Karabel, 1991; Peters, 2003) permiten hacer foco en la dinámica de las tensiones, negociaciones y acuerdos que desarrollan los actores universitarios en la construcción de estrategias institucionales en un entramado institucional que los influye. A partir de este enfoque teórico metodológico, se recupera el proceso de construcción de las estrategias de ingreso de las que participaron en su elaboración diversos actores institucionales. El objetivo de este artículo es analizar la construcción de esas estrategias en la FaHCE, localizando las preocupaciones que dieron origen a esas propuestas, sus características y los cambios que han realizado durante el período 1995-2015. Asimismo, se propone establecer la relación de los lineamientos formulados a nivel central de la Universidad con su expresión en las estrategias estudiadas, y recuperar las perspectivas que sustentan los actores institucionales, los acuerdos y/o tensiones suscitados con relación al procesamiento de esa política. Las fuentes y técnicas de recolección de la información fueron la entrevista cualitativa semi-estructurada en profundidad dirigida a informantes clave y el análisis de documentos institucionales (fuentes estadísticas, estatuto, normativas, disposiciones, programas de ingreso, actas e informes).

En los siguientes apartados se abordará la construcción de las estrategias de ingreso en la FaHCE, distinguiendo dos etapas que se desarrollaron en escenarios disímiles de la política universitaria: los años '90 y a comienzos del nuevo milenio. Las conclusiones retoman los aspectos salientes del análisis desde una perspectiva que considera los aspectos internos y externos para el abordaje de los cambios en materia de acceso a la universidad (Brunner, 1988 citado en Chiroleu, 1999; del Castillo Alemán, 2006). Cabe señalar que el tipo de análisis propuesto se distancia de una explicación teleológica. El estudio realizado no presupone que los propósitos de las estrategias de ingreso hubiesen estado ya contenidos en las marchas y contramarchas del primer período recortado ni que 
hayan ido evolucionando linealmente hacia el actual estado de las cosas, tal como asumiría la filosofía de la historia, según advierte Bourdieu (2011). Más bien, en el análisis de la construcción de la política se muestran las tensiones, las rupturas, los avances y las resistencias de ese proceso, desde una perspectiva multicausal que considera tanto los aspectos internos como externos.

\section{Las estrategias de ingreso en los años '90: entre la masividad y los recursos financieros limitados}

En el año 1991, la FaHCE diseñó un curso de ingreso introductorio para dos de las catorce carreras que brindaba esa UA en aquel momento. Las primeras carreras que optaron por establecer un curso de ingreso fueron Psicología ${ }^{7}$ y Educación Física, cuyas matrículas superaban significativamente a los inscriptos de las demás disciplinas. En efecto, entre fines de los años ' 80 y principio de los ' 90 , se produjo un "aumento incesante de la matrícula”, que tradicionalmente había mantenido niveles reducidos de crecimiento en comparación con otras facultades y un bajo peso en el conjunto de la matrícula de la UNLP. La incorporación de carreras como Psicología y Educación Física fue cambiando paulatinamente aquel perfil (FaHCE, 1992a). Desde la perspectiva institucional, la masificación de las carreras mencionadas quedó consolidada desde el advenimiento democrático, especialmente por la reapertura de la carrera de Psicología en 1984, cerrada en el período 19781983. Esto demuestra que, tras la reapertura democrática de 1983, las políticas de ingreso irrestricto trajeron como consecuencia, por un lado, un incremento exponencial de las matrículas y, por otro, obligaron a las instituciones universitarias a resolver financiera y académicamente el problema de la masificación, con el fin de democratizar el acceso a la educación superior.

Las carreras de Psicología y Educación Física absorbían el mayor porcentaje de la matrícula. En efecto, representaban un $41 \%$ y un $27 \%$, respectivamente del total durante el año 1992, es decir, un $68 \%$ considerada en su conjunto. En el caso de Educación Física, hubo una tendencia de crecimiento desde el año 1984, registrándose entre 1986 y 1989 un pronunciado desarrollo, duplicando y casi triplicando sus ingresos: 357 inscriptos en 1984 a 700 en 1986 y 962 en 1989 (UNLP, 1994). No obstante, se registró una variación entre la cantidad de inscriptos y de aquellos estudiantes que comenzaban a cursar las materias del primer año: 283 ingresantes en 1984, 593 en 1986 y 713 en 1989. Esta situación demostró que el momento inicial de los estudios constituía una primera instancia de selección. En el caso de Psicología, el crecimiento matricular se dio en forma sostenida con dos “picos” de expansión, en 1986 con un 13\% y en 1990, con un 19,6\%, alcanzando un total de inscriptos de 1023 y 1188, respectivamente. Al igual que en Educación Física, la cantidad de ingresantes disminuía considerablemente: 936 ingresantes en 1986 y 983 en 1990. Los documentos institucionales analizaron los procesos de expansión y masificación en ambas carreras e indicaron que "el crecimiento evolutivo de la matrícula no [había] sido acompañado por un crecimiento similar en los egresos” (FaHCE, 1992a, p. 6). En este sentido, y a partir de un análisis de cohortes "estimativas” del año 1984, concluyeron que sólo 16 estudiantes que ingresaban a la FaHCE terminaban sus estudios en el período establecido por el término teórico de su currículo. De este modo, la FaHCE advirtió un "fenómeno de desgranamiento”, al indicar un bajo índice general en la relación ingresos-egresos.

A partir de la situación descripta, la FaHCE instrumentó en el año 1991 un curso introductorio para 
estas carreras, de dos semanas de duración y, en el ciclo lectivo de 1992, se extendió a un mes, con requisito de asistencia. Los actores que impulsaron esa estrategia partieron del reconocimiento de que los aspectos institucionales incidían en el desempeño académico de los estudiantes. Manifestaron que el desgranamiento no sólo se explicaba por los factores externos sobre los que la Universidad no podía actuar de manera directa, sino también a partir de los factores internos. Por tanto, la propuesta impulsada por la Facultad, estuvo centrada en favorecer la integración del estudiante a la vida universitaria, haciendo especial hincapié, por un lado, en los contenidos fundamentales de la carrera elegida y las exigencias propias de sus disciplinas de base y, por el otro, en el conocimiento de la institución universitaria y la reflexión sobre sus características, inserción y transformación. A partir de esta concepción, se diseñaron cursos introductorios asumiendo que los estudiantes debían construir en ese tránsito, diversos saberes que son propios de la disciplina elegida y de la institución universitaria y que, por tanto, requería de un tiempo de aprendizaje específico, que no se demandaba al nivel educativo anterior.

Los procesos de masificación en las carreras citadas fueron mencionados también por la actual Secretaria de Asuntos Académicos y por el Prosecretario de la misma área $\underline{8}$ como problemas que dieron origen a las políticas de ingreso en esa UA. Según los entrevistados, en los orígenes de los cursos introductorios de esa Facultad, la cuestión del ingreso no pudo desligarse del contexto que lo definía como problema, el cual se vinculaba a la masividad y a la falta de recursos financieros. Sobre este último aspecto, los entrevistados coincidieron en señalar que el incremento de la matrícula se produjo en un contexto de crisis económica y presupuestaria de las universidades. Desde las perspectivas relevadas, tanto en los documentos institucionales de la época como en las entrevistas realizadas, la masividad era caracterizada como un "problema” para la institución. En esta clave, la razón que impidió la extensión de los cursos de ingreso a otras carreras ofrecidas por la FaHCE refirió a la cuestión presupuestaria y a condiciones de infraestructura limitadas. Así, la Facultad estableció una estrategia de ingreso para las carreras en las que había una mayor "presión” por ingresar.

A fines del año 1992 en sesiones del Consejo Académico de la FaHCE, los consejeros académicos consideraron oportuno extender la propuesta del ingreso a las demás carreras de la Facultad. Explicitaron la necesidad de establecer un ingreso en las carreras “chicas” debido a que la demanda decrecía cada año (FaHCE, 1992b, p. 2). Como veremos más adelante, estas propuestas se viabilizarán recién en los primeros años del nuevo milenio, a partir de nuevas conceptualizaciones sobre el ingreso, en un nuevo escenario político.

En el año 1994, el Consejo Académico decidió formar una Comisión de Ingreso a fin de cumplir con tareas de diseño y evaluación de los Cursos Introductorios. De esta manera, se institucionalizó la labor de los asuntos del ingreso, a través del funcionamiento de una comisión ad hoc, en la que estaban representados todos los claustros intervinientes. En ese mismo año, se incluyó el diseño y desarrollo de un Plan de Seguimiento y Orientación (POS) dirigido a aquellos estudiantes que no hubieran alcanzado los objetivos del Curso, a fin de brindarles una nueva instancia de acompañamiento y orientación académica, evidenciando así los primeros signos de una política de ingreso que no se agotaba en la duración del Curso, sino que se extendía al tramo del primer año.

A pesar de los cambios introducidos, a fines del año 1993 los miembros de la Comisión de Ingreso 
recomendaron al Consejo Académico, ante una eventual reforma de planes de estudio, incluir un semestre articulatorio o ciclo de nivelación con una modalidad operativa semejante a los cursos introductorios y el desarrollo de actividades similares a las del POS. Esta propuesta de cambio manifestaba una preocupación institucional por extender el tramo del ingreso a un período más extenso, desarrollado dentro de un marco curricular propio de las carreras de grado de esa UA. Finalmente, esta propuesta no logró materializarse dado los recursos limitados que disponía la Facultad para establecer este tipo de cambios.

Respecto del financiamiento de los cursos, en un primer momento la UA los financiaba, aunque con escasos recursos. A partir de 1994, la Facultad presentó la propuesta del ingreso en la convocatoria al Programa de Equiparación de Oportunidades Educativas (PEOE) ${ }^{\underline{9}}$ impulsado por el nivel central, por tanto, accedió a un presupuesto mayor que posibilitó ofrecer a los docentes mejores condiciones de trabajo.

Por su parte, en el Departamento de Lenguas y Literaturas Modernas, la Estrategia de Ingreso era diseñada e implementada por el Departamento docente y financiado por la Facultad. Los alumnos debían presentar una Prueba de Ubicación lingüística correspondiente al idioma respectivo (inglés o francés). El ingreso en ese Departamento se alejaba de los objetivos planteados en los anteriores casos, dado que los estudiantes debían acreditar un nivel de dominio de la lengua para poder cursar las materias específicas. Quienes desaprobaban podían cursar algunas materias en castellano y debían inscribirse en el "Curso de Consolidación de Inglés”. Uno de los testimonios relevados, indicó que en la carrera de Ingles existió una "impronta muy fuerte” acerca de la necesidad de “conocimiento” y "nivel de la lengua”. Para los entrevistados, esta connotación ha sido mantenida durante el período de nuestra investigación, a pesar de los cambios introducidos en la etapa siguiente.

Cabe destacar que, a partir de la sanción de la LES, la cuestión del ingreso fue percibida por los actores institucionales como parte del entramado de políticas de reformas impulsadas por el Estado. En un contexto privatizador y conservador, el ingreso comenzó a interpretarse con rasgos “limitacionistas” propio del clima de época. Por esta razón, veremos que los cambios introducidos en materia de acceso en esta UA, se produjeron en un nuevo contexto social y político.

\section{Las estrategias de ingreso a comienzos del nuevo milenio: cambios y construcción de consensos}

La segunda etapa de construcción de nuevas estrategias de ingreso en la FaHCE se impulsó a mediados de la primera década del 2000. La configuración de un nuevo escenario político en general y universitario en particular, permitió repensar a nivel institucional la perspectiva sobre el ingreso a las carreras de grado. Los entrevistados sostuvieron que, en este período, se redefinió el sentido acerca del ingreso, ya no vinculado al problema de la masividad y a la falta de recursos, sino a un área en la que era posible diseñar una "política educativa” específica.

A partir de la primera década del 2000, estas visiones fueron compartidas con el nivel central considerando que el ingreso se amplió y vinculó con otros lineamientos de la política académica y de bienestar estudiantil orientados a mejorar la integración plena de los estudiantes a la vida universitaria, a través de iniciativas dirigidas al conjunto de los estudiantes, junto con otras 
específicas que focalizan carreras y/o grupos estudiantiles singulares. Entre ellas, se encuentran los programas nacionales generados por la Secretaría de Políticas Universitarias y por el Ministerio de Educación de la Nación, orientados a mejorar la transición entre la escuela secundaria y los ciclos básicos de carreras universitarias. Cabe destacar la sanción de la obligatoriedad del Nivel Secundario consagrada en la Ley de Educación Nacional N 26.206 de 2006, como parte de una serie de cambios en materia educativa iniciados desde 2003, que permitieron ampliar derechos para jóvenes y adultos en nuestro país. Los procesos de cambio señalados coincidieron con la decisión política de la Universidad y el aumento presupuestario impulsados durante los gobiernos kirchneristas (Montenegro, 2016). En efecto, en el período 2003-2014 el presupuesto total de las universidades nacionales ascendió 1.459,36\%, en la UNLP aumentó 1.764,59\%, y en la FaHCE 1.684,79\% durante el mismo período (elaboración propia a partir del Informe Anual Comparado de Indicadores de la UNLP, 2015).

La política institucional de la FaHCE comenzó a conceptualizar la cuestión del ingreso a partir de nuevas coordenadas vinculadas a una “política de ampliación y contención” $\underline{10}$ de los estudiantes. La construcción de esa perspectiva estuvo vehiculizada en parte por las iniciativas que impulsaron los Departamentos de Letras y Ciencias de la Educación, quienes instalaron nuevas perspectivas y espacios institucionales para discutir el ingreso, desde un análisis de las trayectorias estudiantiles y de los procesos de pasaje entre la escuela secundaria y la universidad. Paralelamente, los diversos departamentos docentes retomaron las discusiones para pensar sus propios ingresos, a partir de las especificidades disciplinarias.

El proceso de cambio en la política de ingreso en esta Facultad se inició desde una propuesta y sector específico, en este caso desde las bases operativas del sistema, que lograron ampliar e institucionalizar la discusión en la agenda a través de mecanismos o espacios institucionales. Este proceso permitió arribar a amplios niveles de consenso político sobre los criterios generales acerca del ingreso a nivel de la política de la Facultad y entre los distintos actores de las diversas disciplinas. En este proceso de construcción de políticas, la Facultad otorgó a cada departamento docente la autonomía para definir en “comisiones interclaustro" 11 su propia estrategia aunque fueron conformándose en tiempos diversos, según cada carrera. No obstante, si bien cada departamento elaboraba su propuesta, la Facultad estableció una Comisión General de Ingreso $\underline{12}$ a fin de analizar y evaluarlas y gestionar el financiamiento desde el nivel central. Los rasgos organizacionales de la FaHCE, de tamaño grande y complejo (Balgridge et al, 1973), permiten advertir un alto nivel de autonomía por parte de los departamentos disciplinarios dado que algunas decisiones relativas al ingreso se tomaron de manera descentralizada. El liderazgo departamental fuerte también explicaría la descentralización del trabajo dentro de la estructura compleja de una institución grande. Esta variable manifestaría además la convivencia dentro de esa UA de estrategias de ingreso con propósitos no necesariamente coincidentes.

Una de las discusiones generadas en esta segunda etapa refirió a la cuestión de la obligatoriedad en la asistencia de los cursos, aunque con matices según las carreras. A partir de las lógicas disciplinares, de la historia y cultura de los departamentos, la discusión sobre el tipo de carácter del curso estuvo vinculada a la idea de evitar que fueran “potencialmente restrictivos”. Por estas razones, los diversos cursos fueron adoptando rasgos diversos en su carácter, tanto obligatorios 
como optativos.

Se identificaron características que prevalecieron en las estrategias de ingreso como parte de los consensos construidos. La mayoría fueron de carácter introductorio y se diseñaron a partir de la especificidad disciplinar. Al respecto, desde la perspectiva de los actores, si bien en los cursos de ingreso se abordaron algunos contenidos específicos, no se plantearon desde la carencia, de aquello que no "sabían” los estudiantes. Más bien, se propuso desarrollar nuevos saberes sobre la lectura y escritura requeridas para participar en las culturas discursivas de las disciplinas. Así, se abocaron a trabajar la alfabetización académica, en tanto se concibió que la universidad debe enseñar lo que la institución exige, dado que esos conocimientos se consideraban "nuevos” y “distintos”. En tal sentido, se planteó como rasgo común, contribuir a la formación del oficio de estudiante universitario. Otra de las características refirió a la ambientación universitaria, a fin favorecer la integración institucional a partir del conocimiento de sus reglas. Al mismo tiempo, las estrategias también permitían fomentar la sociabilidad entre pares y docentes, construir vínculos y maneras de habitar la institución, aspectos que son considerados como variables que intervienen en la integración del estudiante a la vida universitaria.

La configuración de las nuevas estrategias de ingreso, en esta segunda etapa, se expresaron como una forma básica del cambio, por no contar con una antigüedad mayor en su implementación (Clark, 1983). En este sentido, las carreras que no tenían cursos de ingreso contaron con poca estructura y culturas propias para conducir la interacción y el cambio. Pero, en la medida en que fueron desarrollándose, los procesos de diálogo y de construcción de consenso favorecieron a la definición de su propia identidad sustentada por concepciones y racionalidades compartidas por los actores que participan y operan en ese marco institucional.

Con relación a la vinculación de la Facultad con el nivel central, los actores entrevistados señalaron que la construcción de consensos acerca del ingreso entre las UA y la Universidad se alcanzó a partir del conflicto con la Facultad de Ciencias Médicas a causa de su sistema de acceso restrictivo. Al mismo tiempo, la reforma del Estatuto en 2008 permitió establecer y "reforzar" un criterio básico sobre un sistema de ingreso en la UNLP, a partir de su pronunciamiento como "libre e irrestricto”. En lo que refiere a principios, esta norma reformada indicó el establecimiento explícito de la gratuidad de los estudios de grado y estableció el ingreso irrestricto de carácter libre. La introducción de principios de gratuidad, ingreso irrestricto y derecho a la educación superior, puede entenderse como una forma de clausurar las pretensiones de arancelamiento y selección que la LES había dejado abiertas.

Es posible afirmar que, durante el período estudiado, se configuraron estrategias de ingreso orientadas a mejorar la inserción plena y el acceso efectivo a los estudios universitarios. En efecto, los entrevistados sostuvieron que las estrategias fueron pensadas como "un arco" que permitiese alcanzar a todo el primer año aunque consideraron que la articulación efectiva de las prácticas del primer año con el ingreso es un objetivo "muy ambicioso". No obstante, señalaron que han desarrollado estrategias con las cátedras del primer año, vinculadas al abordaje de la permanencia de los estudiantes. Desde la perspectiva institucional, el ingreso involucra tanto el acceso como la permanencia de los estudiantes a la vida universitaria, por tanto, conciben que las materias de los primeros años deben acompañar ese "trayecto escalonado", combinando el nivel académico con 
mecanismos de apoyo y contención. Respecto a esta visión, se asume un fuerte énfasis en impulsar cambios en las estructuras curriculares y en las prácticas de enseñanza, en particular, en las asignaturas del primer año, a fin de conmover la matriz tradicional de la universidad.

\section{Conclusiones}

La investigación en la que se enmarca este trabajo asumió que la adopción de una determinada política de ingreso a la universidad es un proceso complejo en cuya configuración intervienen los factores internos y externos. $\frac{13}{}$ La política de ingreso a los estudios universitarios es una construcción histórica y tiene vinculación con las políticas sociales nacionales. Por tanto, los cambios en materia de acceso del nivel universitario no se dan en el vacío sino que obedecen a una cierta lógica y a los límites establecidos por las formas históricas de relacionamiento de los agentes intervinientes.

El estudio realizado posibilitó revisar el supuesto de partida, al mostrar que los cambios introducidos a nivel local en materia de ingreso fueron previos a la sanción de la LES. Los procesos de masificación en las carreras de grado tras la reapertura a la democracia en nuestro país, y los altos índices de desgranamiento, constituyeron "problemas" que ingresaron en la agenda institucional y que demandaron el establecimiento de un curso de ingreso para las carreras masivas de esa Facultad, a partir del año 1991. Este resultado puede ser explicado por los procesos de cambio que a nivel nacional se impulsaron en las universidades públicas en el período de la postdictadura. En ese escenario, el juego político que se desarrolló entre el Estado Nacional y las universidades marcó una redefinición en la orientación de las políticas nacionales de acceso a la educación superior, que habilitó la apertura y consiguiente masificación universitaria, tras un período de restricciones y achicamiento del sistema.

La reconstrucción realizada permitió comprender que la FaHCE estableció su curso de ingreso previo a la creación del PEOE impulsado por el nivel central de la Universidad en 1994. Este resultado es consistente con los planteos de las perspectivas organizacionales y de la sociología de la cultura, en el sentido que se evidencia el nivel de autonomía relativa que tuvieron los actores institucionales para la elaboración de un curso de ingreso, con antelación a la definición de una política, tanto a nivel nacional, como en el ámbito de la UNLP.

Los hallazgos mostraron que la cuestión del ingreso se caracterizó como “problema” en un contexto específico de la política universitaria impulsada en los años '90. En un escenario de profundas reformas y redefiniciones entre el Estado y la universidad, se instaló el modelo del "Estado evaluador”, vinculado a la rendición de cuentas por parte de las instituciones universitarias. Por tanto, las universidades se convirtieron en blanco de una serie de disputas, entre las cuales el ingreso a sus aulas ocupó un lugar de importancia (Chiroleu, 1999). A partir de la sanción de la LES, el ingreso formó parte del entramado de políticas de reformas impulsadas por el Estado. En este contexto político, se cuestionaba la legitimidad del sistema universitario abierto, signado por el ingreso irrestricto y la gratuidad, en el marco de las ideas neoliberales sobre la eficacia del gasto en educación. En este plano de interpretaciones, los altos índices de “desgranamiento” generaron preocupación al interior de las instituciones, por lo que el ingreso en la FaHCE fue incluido en la construcción de una estrategia que se orientó a dar respuesta a ese problema, en un contexto de 
recursos financieros limitados.

A partir del nuevo milenio, se establecieron cambios en la orientación de las políticas de ingreso impulsadas desde el Estado Nacional. Se incorporaron a la agenda universitaria, diversas estrategias orientadas a mejorar la transición entre la escuela secundaria y los ciclos básicos de carreras universitarias, impulsadas desde ámbitos centrales y locales de formulación de políticas. La redefinición de la política de ingreso a nivel nacional desde el año 2003 fue acompañada de un incremento de los recursos financieros que posibilitaron la concreción de líneas de acción que ampliaron y complejizaron la conceptualización de las políticas de ingreso. Si bien, durante los gobiernos kirchneristas la política universitaria no logró instalar una agenda de cambios para el sector que estuviera orientada a revisar el legado proveniente de los años '90 y a elaborar una política universitaria integral (Chiroleu e Iazzetta, 2012), los resultados mostraron que esos cambios sucedidos desde la política nacional contribuyeron a redefinir el sentido de la política de ingreso implementada a nivel local. Esto significó un cambio en las perspectivas caracterizado por el pasaje de una demanda de ampliación del acceso a una política de ingreso que contemple además la permanencia y el egreso.

Desde una mirada internista, las caracterizaciones realizadas pusieron en evidencia la complejidad que asumen los procesos de cambio en las políticas y estrategias de ingreso de las universidades como organizaciones de base pesada y con una cierta renuencia a las transformaciones profundas. Los hallazgos mostraron que, más que definiciones que bajan desde las estructuras organizativas, los cambios se terminan gestando "desde abajo". Muchas veces los procesos de cambio resultan sustentables cuando los impulsan grupos académicos particulares, con intereses que confluyen en una decisión compartida. Así, los procesos de cambio en la universidad, entendida como un sistema complejo de toma de decisiones y arreglos de poder, difícilmente puedan ser impuestos o generados desde la cúpula universitaria sin persuadir o generar coaliciones en los grupos localizados en las unidades operativas (Krotsch, 2001). En efecto, el proceso de cambio en la FaHCE se inició desde la base operativa del sistema, que logró ampliar e institucionalizar la discusión en la agenda a través de mecanismos institucionales. Este proceso permitió arribar a consensos sobre los criterios generales acerca del ingreso a nivel de la política de la Facultad y entre los distintos actores de las diversas disciplinas. Los enfoques neoinstitucionalistas permiten mostrar que más allá de las variables organizacionales, los actores universitarios tienen autonomía relativa para negociar, resistir, adecuarse, acordar entre sí y con otros sectores de las UA o de la Universidad, al momento de definir las estrategias de ingreso, en un marco institucional en el que operan.

Se identificó que las orientaciones de las estrategias de ingreso en el período estudiado fueron definiéndose desde una mirada cada vez más integral y compleja, concibiendo al ingreso como un momento peculiar en el que el estudiante debe construir saberes específicos que son propios del oficio del estudiante universitario. Así, se fue configurando una tendencia que pasó de concebir al ingreso como un período acotado de un mes a entenderlo como parte de la dinámica misma del primer año, con propuestas niveladoras e instancias de orientación y tutoría que se desplegaron a lo largo del ciclo académico, aunque como instancias alternativas. Durante la segunda etapa, se registraron de manera incipiente perspectivas que asumieron que, además de una temporalidad más extendida, las estrategias de ingreso deben articularse con cambios que, a nivel curricular es preciso introducir, sobre todo, en las materias del primer año. En esta mirada se registra un énfasis puesto 
en introducir cambios en la enseñanza, y sobre todo en el currículo.

La indagación mostró que en esta UA se diseñaron estrategias inclusivas tendientes a promover la igualdad de oportunidades para todos los estudiantes de las carreras de grado. La concepción de ingreso en esta Facultad es consistente con las orientaciones estratégicas definidas a nivel central de la UNLP y esa coincidencia se expresó además en los criterios generales consensuados para la elaboración de los programas de ingreso. En este sentido, tanto la Universidad como la Facultad suscriben políticas de acceso coherentes en cuanto a la inclusión de los ingresantes a la vida universitaria. No obstante, como vimos, encontramos estrategias con propósitos no necesariamente coincidentes. En este caso, la cuestión de la exigencia de un nivel del idioma también daría cuenta de las formas en que la disciplina contribuye a la formación requerida para el desempeño profesional. Esta variable introduce nuevas tensiones en el proceso de definición de una política: el mercado laboral demandaría profesionales con alto nivel de dominio de una lengua extranjera que podría quedar asociado a una política de ingreso selectiva y restrictiva; mientras que, la Facultad plantea estrategias y modalidades que permitan el ingreso a ese tipo de carreras, aún para aquellos estudiantes que no hubieran alcanzado la suficiente formación previa del idioma, con antelación a su ingreso a la carrera de grado.

\section{Notas}

1 Agradezco especialmente a Luciana Garatte y Gabriela Marano por sus valiosos aportes a la mejora de este artículo.

$\underline{2}$ La investigación citada se realizó en el marco de una Beca tipo A otorgada por la UNLP e inscripta en una Tesis de maestría en Educación por la UNLP.

$\underline{3}$ Las palabras entrecomilladas aluden a una categoría nativa establecida por la UNLP para denominar las propuestas de ingreso que cada UA establece para la incorporación de los estudiantes a las carreras de grado.

4 Las Facultades elegidas fueron Ciencias Exactas, Ciencias Médicas, Ciencias Agrarias y Forestales y Humanidades y Ciencias de la Educación.

\section{$\underline{5}$ Informe Anual Comparado de Indicadores de la UNLP, 2015.}

$\underline{6}$ Se considera carreras "pequeñas” a aquellas que no superan los 200 ingresantes (Ciencias de la Educación, Bibliotecología, Filosofía, Letras, Geografía, Sociología, entre otras); las “medianas” no superan los 500 ingresantes (Traductorado de Inglés e Historia) y las carreras "grandes” superan los 1000 ingresantes (Educación Física). Para establecer esta clasificación, se tomaron los datos de las matrículas de ingresantes extraídos de FaHCE, 2016.

$\underline{7}$ En aquellos años, la carrera de Psicología formaba parte de esa UA, la cual se insertaba en el Departamento de Psicología. En el año 2006 se creó la Facultad de Psicología en la UNLP. Con relación a las implicancias de la salida de esa carrera en la FaHCE, se considera un aspecto a profundizar dado que excedió los límites de esta indagación. No obstante, se podrían formular algunas hipótesis al respecto. Por un lado, la evolución del presupuesto de la FaHCE indicaría que 
los recursos económicos fueron acrecentándose, pasando de un total de 16.845.661,24 pesos, en 2006 a 20.408.634 pesos, en 2007 (UNLP, 2008). Los datos mostrarían que en materia presupuestaria, la FaHCE no sufrió reducción de recursos por la salida de Psicología, por el contrario, fueron acrecentados. En tal sentido, se podría afirmar a modo de hipótesis que, la retirada de esta carrera en la FaHCE descomprimió la asignación de recursos financieros, lo cual posibilitó redistribuir los recursos económicos a las carreras "chicas" que durante la primera etapa no contaban con programas de ingreso. Por otro lado, se podría afirmar que la ausencia de esta carrera masiva pudo haber incidido en el cambio de direccionalidad de la política de ingreso, ya no centrada en aspectos vinculados estrictamente a la masividad, sino a la retención de matriculas exiguas en las demás carrera de esa UA.

$\underline{8}$ Ambos fueron entrevistados el 29 de mayo de 2015 en la Ciudad de La Plata.

$\underline{9}$ Se trató de un Programa impulsado por la UNLP que tuvo como principales objetivos desarrollar instancias articuladoras entre niveles del sistema educativo y retener matrícula en el tramo inicial de las carreras. En 1994 la Secretaría de Asuntos Académicos de la UNLP a través del PEOE, buscó enmarcar y nuclear las propuestas de cada una de las Facultades en una política institucional más amplia, a fin de regular y establecer criterios comunes en las estrategias de ingreso desarrolladas por las UA (Montenegro, 2016).

10 Las palabras entrecomilladas en este párrafo y en los siguientes corresponden a expresiones textuales de los entrevistados.

11 Se trató de una comisión de ingreso ad hoc, integrada por un jefe de departamento y por los representantes de profesores, graduados y estudiantes.

12 Desde el año 2006, esta Comisión se constituyó en un espacio de elaboración de políticas de ingreso coordinado por la Secretaría de Asuntos Académicos de la Facultad, en el que participaban directores de los Departamentos docentes y miembros de las comisiones de ingreso.

13 Por razones de extensión y alcance del presente trabajo, sólo se presentarán algunos aspectos de la incidencia de los factores externos en el análisis del caso. Para profundizar este aspecto ver Montenegro, 2016.

\section{Fuentes consultadas}

FaHCE, UNLP (1992a). Aproximaciones a una reflexión sobre el ingreso a la Universidad desde la perspectiva de articulación entre el nivel secundario y el terciario. La Plata. Mimeo.

FaHCE, UNLP (1992b). Acta n¹12, 06/08/1992. Mimeo.

FaHCE, UNLP (1993a). Evaluación Ingreso 1993 a la FaHCE de la UNLP y Proyecto Ingreso 1994. La Plata, Mimeo.

FaHCE, UNLP (2016). Ingresantes 2016 comparado 2015. Dirección de Enseñanza. La Plata, Mimeo.

UNLP (1994a). Secretaria de Asuntos Académicos. Origen socioeducativo de los ingresantes y 
rendimiento académico. La Plata, Mimeo.

UNLP. (2008). Informe Anual Comparado de Indicadores de la UNLP. Disponible en http://www.unlp.edu.ar/uploads/docs/anuario indicadores2008.pdf

UNLP. (2015). Informe Anual Comparado de Indicadores de la UNLP. Disponible en: http://www.unlp.edu.ar/articulo/2011/11/17/anuario de indicadores 2015

\section{Bibliografía}

Balgridge, J. V., Curtis, D., Ecker, G. \& Riley, G. (1973). The impact of institutional size and complexity on Faculty Autonomy [El impacto del tamaño institucional y la complejidad en la autonomía de la facultad]. The Journal of Higher Education, 44(7), 532-547.

Bracchi, C., Sannuto, J. y Mendy, M. (2002). Políticas de Educación Superior: el ingreso a los estudios universitarios en la UNLP. En P. Krotsch, (organizador), La universidad cautiva: Legados, marcas y horizontes. La Plata: Al Margen.

Becher, T. (2001). Tribus y territorios académicos. La indagación intelectual y las culturas de las disciplinas. Barcelona: Gedisa.

Brint, S. y Karabel, J. (1991). Los orígenes y las transformaciones institucionales: el caso de las escuelas locales de los Estados Unidos. En W. Powel y P. DiMaggio (eds), El nuevo institucionalismo en el análisis organizacional (pp. 413-440). México: Fondo de la Cultura.

Bourdieu, P. (1983). Campo del poder y campo intelectual. Buenos Aires: Folios.

Bourdieu, P. (2008). Homo academicus. Buenos Aires: Siglo XXI.

Bourdieu, P. (2011). La ilusión biográfica. Acta Sociológica, 56, 121-128.

Del Castillo Alemán, G. (2006). Una propuesta analítica para el estudio del cambio en las instituciones de educación superior. Perfiles Educativos, XXVIII(111), 37-70.

Duarte, B. (2009). De aspirantes a alumnos. Una clasificación de los sistemas universitarios de admisión y su aplicación a las universidades nacionales de la Argentina. En S. Gvirtz y A. Camou (coord.), La universidad Argentina en discusión: sistemas de ingreso, financiamiento, evaluación de la calidad y relación universidad-Estado (pp. 27-58). Buenos Aires: Granica

Chiroleu, A. (1999). El ingreso a la Universidad: las experiencias de Argentina y Brasil. Rosario: UNR Editora.

Chiroleu, A. y Iazzetta, O. (2012). La universidad como objeto de política pública durante los gobiernos Kirchner. En A. Chiroleu, M. Marquina y E. Rinesi. (comps.), La política universitaria de los gobiernos Kirchner: continuidades, rupturas, complejidades (pp. 19-39).Los Polvorines: Universidad Nacional de General Sarmiento.

Clark, B. (1983). El sistema de educación superior. Una visión comparativa de la organización académica. México: Universidad Autonomía Metropolitana. 
Cox, C. (1993). Políticas de educación superior: categorías para su análisis. En H. Courard, (edit.). Políticas Comparadas de Educación Superior en América Latina (pp. 97-129). Santiago de Chile: FLACSO.

Krotsch, P. (2001). Educación Superior y Reformas Comparadas. Cuadernos Universitarios Nº 6. Buenos Aires: UNQUI Ediciones.

Montenegro, J. (2016). Políticas de acceso a la Universidad Nacional de La Plata. Un análisis de las estrategias de ingreso desde la sanción de la Ley de Educación Superior (1995-2015). Tesis de maestría, Facultad de Humanidades y Ciencias de la Educación, Universidad Nacional de La Plata, Ensenada, Argentina. Disponible en: http://sedici.unlp.edu.ar/handle/10915/57617

Peters, G. (2003). El nuevo institucionalismo. Teoría institucional en ciencia política. España: Editorial Gedisa.

Ramallo, M. y Sigal, V. (2010). Los sistemas de admisión de las Universidades en la Argentina (255). Recuperado de Universidad de Belgrano: http://www.ub.edu.ar/investigaciones/dt nuevos/255 sigal.pdf 